\title{
A!
}

This is an electronic reprint of the original article.

This reprint may differ from the original in pagination and typographic detail.

Mäki, J.-M.; Tuomisto, F.; Varpula, Matti; Fisher, D.; Khan, R.U.A.; Martineau, P.M.

\section{Time dependence of charge transfer processes in diamond studied with positrons}

Published in:

Physical Review Letters

DOI:

10.1103/PhysRevLett.107.217403

Published: 01/11/2011

Document Version

Publisher's PDF, also known as Version of record

Please cite the original version:

Mäki, J-M., Tuomisto, F., Varpula, M., Fisher, D., Khan, R. U. A., \& Martineau, P. M. (2011). Time dependence of charge transfer processes in diamond studied with positrons. Physical Review Letters, 107(21), 1-5. [217403]. https://doi.org/10.1103/PhysRevLett.107.217403

This material is protected by copyright and other intellectual property rights, and duplication or sale of all or part of any of the repository collections is not permitted, except that material may be duplicated by you for your research use or educational purposes in electronic or print form. You must obtain permission for any other use. Electronic or print copies may not be offered, whether for sale or otherwise to anyone who is not an authorised user. 


\title{
Time Dependence of Charge Transfer Processes in Diamond Studied with Positrons
}

\author{
J.-M. Mäki* and F. Tuomisto \\ Department of Applied Physics, Aalto University, P.O. Box 11100, FI-00076 Aalto, Espoo, Finland \\ A. Varpula \\ Department of Micro and Nanosciences, Aalto University, P.O. Box 13500, FI-00076 Aalto, Espoo, Finland \\ D. Fisher, R. U. A. Khan, and P. M. Martineau \\ DTC Research Center, Belmont Road, Maidenhead, Berkshire SL6 6JW, United Kingdom
}

(Received 8 July 2011; published 16 November 2011)

\begin{abstract}
We have developed a method called optical transient positron spectroscopy and apply it to study the optically induced carrier trapping and charge transfer processes in natural brown type IIa diamond. By measuring the positron lifetime with continuous and pulsed illumination, we present an estimate of the optical absorption cross section of the vacancy clusters causing the brown color. The vacancy clusters accept electrons from the valence band in the absorption process, giving rise to photoconductivity.
\end{abstract}

Positron annihilation spectroscopy is an experimental method sensitive to neutral and negatively charged vacancy defects in semiconductors [1]. Measurements performed at varying temperatures have revealed many important properties of charged defects such as the Ga vacancies in $\mathrm{GaN}$ [2] or the $\mathrm{Zn}$ vacancies in $\mathrm{ZnO}$ [3]. Combining traditional ways of measuring with optical illumination during measurements has led to identifying, e.g., the monovacancy character of the midgap electron trap (EL2) defect in GaAs [4] and the donor-complex (DX) center in $\mathrm{Al}_{x} \mathrm{Ga}_{1-x} \mathrm{As}$ [5]. More recently, similar experiments have been used to identify the contribution of $V_{\mathrm{Si}}$ behind some of the optical transitions in $\mathrm{SiC}$ [6], in addition to identifying the origin of the brown coloration of highest purity natural diamond $[7,8]$. The sensitivity to the charge state (observed as an enhanced signal from negative centers especially at low temperatures) is the key feature of the positron methods when optical illumination is applied, as the defect levels can be populated or depopulated by photon absorption.

We have developed a method to perform positron measurements during the relaxation of optically excited semiconductors, called optical transient positron spectroscopy. We obtain information on the energy levels of the vacancy defect(s), their concentrations, and carrier capture and recombination rates. Correlating with photoconductivity measurements gives the possibility to analyze also the behavior of photogenerated free carriers. To demonstrate the potential of the developed method, we apply it to brown type IIa diamond that exhibits colossal effects on positron data during illumination [8]. However, the method is not limited to studying defects in natural diamond. For example, the dynamic optical properties of synthetic diamond are of considerable interest in light of potential applications in high power solid state optoelectronics
$[9,10]$. The advances in the chemical vapor deposition (CVD) of diamond $[11,12]$ and the long spin decoherence times of nitrogen-vacancy $(\mathrm{N}-V)$ centers $[13,14]$ have recently brought significant pressure into obtaining a better understanding of the charge transfer processes in diamond. Reports on the observation of monovacancy defects in type Ib CVD diamond exist $[15,16]$, and $\mathrm{N}-V$-related centers have been shown to exhibit optical activity with positron measurements [17].

We present positron lifetime results on the timedependent photoexcitation of vacancy type defects in natural type IIa (highest purity, less than $10^{16} \mathrm{~cm}^{-3}$ nitrogen) diamond. The measured sample pair shows a slight brown color resulting from a continuous absorption ramp starting at $2.0 \mathrm{eV}$, typical of brown diamond [18]. The defect behind the brown coloration has been identified as a vacancy cluster of 40-60 missing atoms [8] producing a lifetime component of about $400 \mathrm{ps}$, supported by theoretical considerations and experiments [19,20]. Optical sub-band-gap excitation results in negative charging of the vacancy cluster, causing the visible absorption. Brown type IIa diamond is used to demonstrate the method as the 400 ps positron lifetime is clearly visible in the experimental spectrum, enabling us to concentrate on the effects of illumination. More subtle changes in the positron annihilation data (such as those produced by monovacancy defects) can be studied as well, as long as the charge state of the vacancies of interest can be manipulated by optical excitation [4-6,21].

The positron lifetime was recorded by using conventional instrumentation [1] with a possibility to illuminate the samples [22]. The lifetime spectrum is analyzed as a superposition of exponential decay components $n(t)=$ $\Sigma_{i} \exp \left(-t / \tau_{i}\right)$ convoluted with a Gaussian resolution function. The positron in state $i$ annihilates with lifetime $\tau_{i}$ and 
intensity $I_{i}$. The spectrum can be decomposed to 1-3 components corresponding to annihilations in a delocalized state in the lattice or a localized state at vacancies. The increase of average lifetime $\tau_{\text {ave }}=\Sigma_{i} I_{i} \tau_{i}$ above the lifetime in bulk material is an indication of vacancy (open volume) defects being present. The transition rate for the thermalized positron to a vacancy (called the trapping rate) is defined as $\kappa=\mu_{D} c_{D}$, where $c_{D}$ is the defect concentration and $\mu_{D}$ is the trapping coefficient.

High power light emitting diodes (LEDs) in near-UV, blue, and green wavelengths were used for the optical illumination. The near-UV LED had nominal radiant flux of $700 \mathrm{~mW}( \pm 5 \%)$ at wavelength $400 \mathrm{~nm}(3.1 \mathrm{eV})$ ( $\pm 5 \%$ ), the blue LED $970 \mathrm{~mW}$ (at $2.7 \mathrm{eV}, 465 \mathrm{~nm}$ ), and the green $340 \mathrm{~mW}(2.4 \mathrm{eV}, 525 \mathrm{~nm})$. Collecting the positron lifetime spectra was started after switching off the light source, and the spectrum was recorded in slots of $1-10 \mathrm{~s}$ so that the positron lifetime spectra were obtained at times 5, 15, $25 \mathrm{~s}$, etc., after the illumination. The slot length did not affect the positron lifetime results. Photoconductivity was studied by using the same LEDs.

We propose a simple two-state model for analyzing the effect of the optical excitation. Based on the earlier results of positron measurements as a function of illumination and temperature $[7,8]$, it is clear that electrons are excited into the originally neutral vacancies that become negatively charged. By assuming that electrons are excited directly from the valence band to the vacancy cluster, the transition rate can be written as

$$
\frac{d\left[V^{-}\right]}{d t}=\sigma \phi\left[V^{0}\right]-g\left[V^{-}\right]
$$

where $\left[V^{0}\right]$ and $\left[V^{-}\right]$are the concentrations of vacancies in neutral and negative charge states, respectively, $\sigma$ is the optical absorption cross section of the vacancy cluster, $\phi$ is the photon flux, and $g$ is the transition rate back to the neutral charge state. During steady-state illumination, electrons are excited to the vacancy cluster with the same rate as holes and electrons recombine, i.e., $\frac{d\left[V^{-}\right]}{d t}=0$. Therefore, the fraction of clusters with single negative charge can be written as

$$
\frac{\left[V^{-}\right]}{\left[V_{\text {dark }}^{0}\right]}=\frac{\phi}{\phi+g_{s} / \sigma}
$$

by taking into account the conservation of vacancy concentration $\left[V_{\mathrm{dark}}^{0}\right]=\left[V^{-}\right]+\left[V^{0}\right]$ and by assuming the transition rate $g_{s}$ to be constant in the steady state.

The average positron lifetime $\tau_{\text {ave }}$ as a function of photon flux is plotted in the upper panel of Fig. 1 when illuminated with a $3.1 \mathrm{eV}$ LED at 200 and $300 \mathrm{~K}$, with $2.7 \mathrm{eV}$ at $200 \mathrm{~K}$, and with $2.4 \mathrm{eV}$ at $300 \mathrm{~K}$. $\tau_{\text {ave }}$ behaves in a similar manner irrespective of the illumination wavelength or temperature. $\tau_{\text {ave }}$ starts to increase above fluxes $10^{13} \mathrm{~cm}^{-2} \mathrm{~s}^{-1}$, and $\tau_{\text {ave }}$ saturates when the flux is close

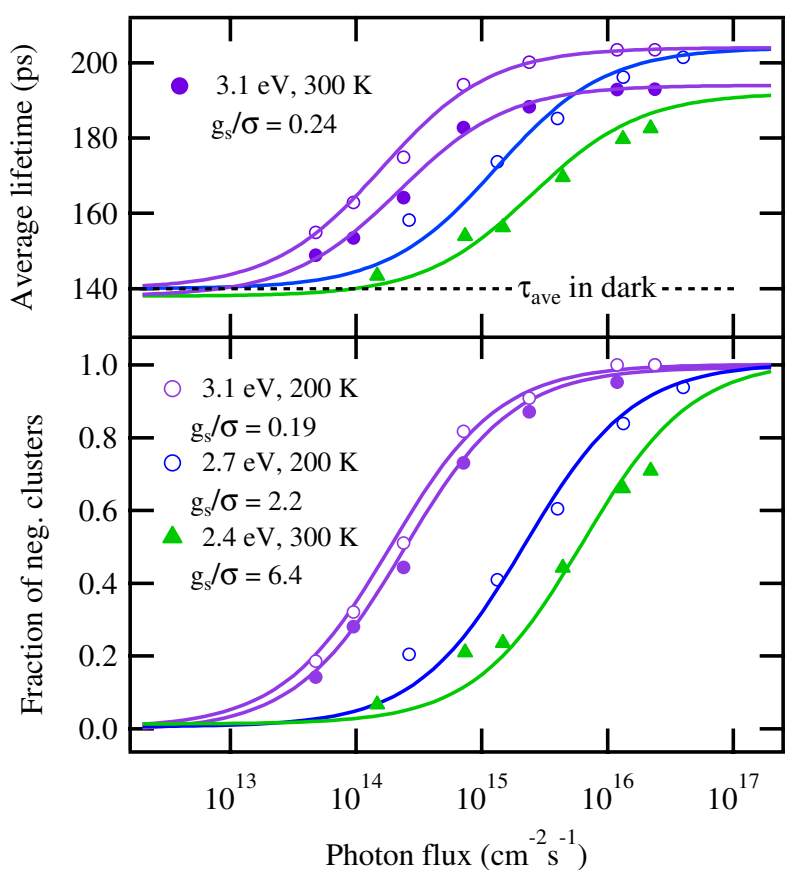

FIG. 1 (color online). $\tau_{\text {ave }}$ (upper panel) and the fraction of negative clusters estimated from Eq. (3) (lower panel) as a function of illumination flux. The excitation wavelength and the measurement temperature are indicated. The ratio $g_{s} / \sigma$ is fitted according to Eq. (2) and is given in units $10^{15} \mathrm{~cm}^{-2} \mathrm{~s}^{-1}$.

to $10^{17} \mathrm{~cm}^{-2} \mathrm{~s}^{-1}$. The saturation value of $\tau_{\text {ave }}$ depends on the temperature dependence of the trapping to the vacancy cluster; i.e., the trapping coefficient of the negative cluster slightly decreases between 200 and $300 \mathrm{~K}$. $\tau_{\text {ave }}$ increases as a function of flux due to the fact that more vacancy clusters become negatively charged, attracting more positrons due to the Coulomb attraction driven increase in the trapping coefficient. We assume that $\tau_{\text {ave }}$ saturates with $2.4 \mathrm{eV}$ illumination to the same values as for 3.1 and $2.7 \mathrm{eV}$; i.e., all neutral vacancy clusters could be converted into negative if the flux is high enough.

The exact charge state of the defect cannot be deduced, as the difference between the different negative states is expected to be much smaller than the difference between neutral and negative [23]. Hence, most of the effect detected as a function of flux is due to the disappearance of neutral clusters, and the saturation of $\tau_{\text {ave }}$ can be attributed to none of the clusters being in a neutral charge state; i.e., the measurement is primarily sensitive to the number of neutral clusters.

The fraction of clusters in the negative charge state can be written as

$$
\frac{\left[V^{-}\right]}{\left[V_{\mathrm{dark}}^{0}\right]}=\frac{\kappa_{2}-\kappa^{0}}{\kappa^{-}-\kappa^{0}}
$$

where $\kappa_{2}$ is the trapping rate to the vacancy clusters, $\kappa_{0}$ is the trapping rate to the neutral cluster (in the dark), and $\kappa^{-}$ 
is the trapping rate when the positron lifetime has saturated (all clusters in a negative charge state). $\kappa_{2}$ has been estimated by using the trapping model shown in detail in Refs. [8,24]. The fraction of clusters in a negative charge state is shown in the lower panel of Fig. 1 along with a fit of $g_{s} / \sigma$ based on the model described above [Eq. (2)] measured at either 200 or $300 \mathrm{~K}$. The fit corresponds to the experimental data very well.

The decay rate $g$ of the optical excitation must be solved in order to obtain the optical absorption cross section $\sigma$ of the defect. This is done by solving Eq. (1) after switching off the illumination. The hole capture rate from the valence band to the electron level corresponding to the vacancy cluster can be written as $g=\sigma_{p}\left\langle v_{p}\right\rangle p$, where $\sigma_{p}$ is the hole capture cross section, $\left\langle v_{p}\right\rangle$ is the thermal velocity, and $p$ is the concentration of free holes. We assume that at and below room temperature the majority of free holes is caused by photoexcitation so that $p=\left[V^{-}\right]$for singly negative vacancy clusters. By solving the transition rate, the concentration of the negative clusters as a function of time is

$$
\left[V^{-}(t)\right]=\frac{\left[V_{s}^{-}\right]}{\sigma_{p}\left\langle v_{p}\right\rangle\left[V_{s}^{-}\right] t+1},
$$

where $\left[V_{s}^{-}\right]$is the concentration of negative clusters before switching the illumination off (in the steady state).

Figure 2 shows positron annihilation spectra at selected times after switching off the illumination. The measurement was cycled 1000 times in order to obtain sufficient annihilation statistics for the final spectra. It is clear that the lifetime component $\tau_{2}=400 \mathrm{ps}$ remains unchanged, but the intensity of the component decreases as a function of time resulting in the decrease of $\tau_{\text {ave }}$. Thus, $\tau_{\text {ave }}$ is governed by the intensity of the 400 ps lifetime component as in the steady-state measurements. The spectra can be fitted with two lifetime components, and the

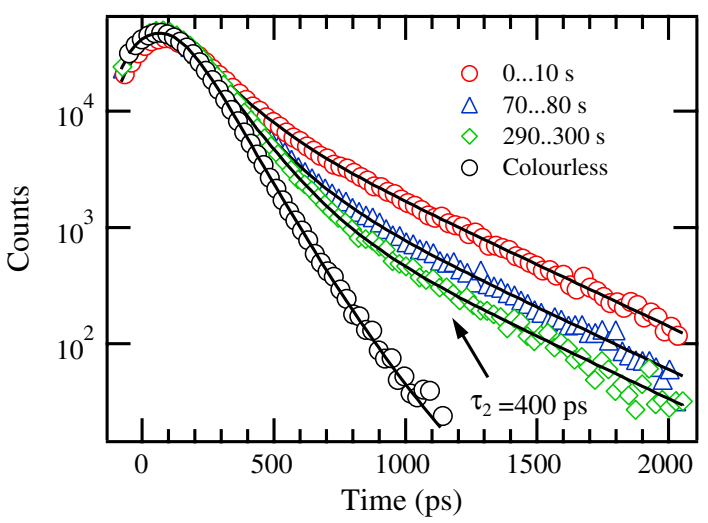

FIG. 2 (color online). The positron lifetime spectra obtained after $3.1 \mathrm{eV}$ illumination at room temperature. The labels correspond to time in the dark after switching off the illumination. A lifetime spectrum of a colorless diamond is shown for comparison. trapping rate to the vacancy clusters can be estimated as $\kappa_{2} \propto I_{2} / I_{1}[8]$.

The fraction of negative clusters after the illumination can be estimated in a similar manner as in the steady state. The experimental points determined from the trapping rates are shown in Fig. 3 along with the fit based on Eq. (4). The decay spectra show only a single decay constant $g_{s}$, indicating the presence of only one (or one dominating) recombination path.

We also studied the flux dependence of the decay of the photoexcitation from $10^{14}$ to $10^{16} \mathrm{~cm}^{-2} \mathrm{~s}^{-1}$ at and below room temperature. Interestingly, the transition rate $g$ is constant for a given photon energy within the measurement precision irrespectively of the concentration of negative clusters at $t=0$, justifying the constant $g$ in the model for the steady-state illumination. The functional form of $g$ hence indicates that the hole capture cross section is inversely proportional to the vacancy cluster concentration or that the free hole concentration increases less rapidly than the negative vacancy cluster concentration. The exact origin of this behavior remains to be clarified.

The determined optical absorption cross sections (they increase with photon energy as can be expected from the typical absorption coefficient of brown type IIa diamond [8]) are shown in Fig. 4 together with the optical absorption coefficient $\alpha$. The optical absorption cross section was measured also at several measurement temperatures down to $45 \mathrm{~K}$ (not shown) with estimated values being similar as in RT. We performed the flux- and wavelengthdependent experiments also on high temperature annealed brown diamond where the vacancy cluster concentration is

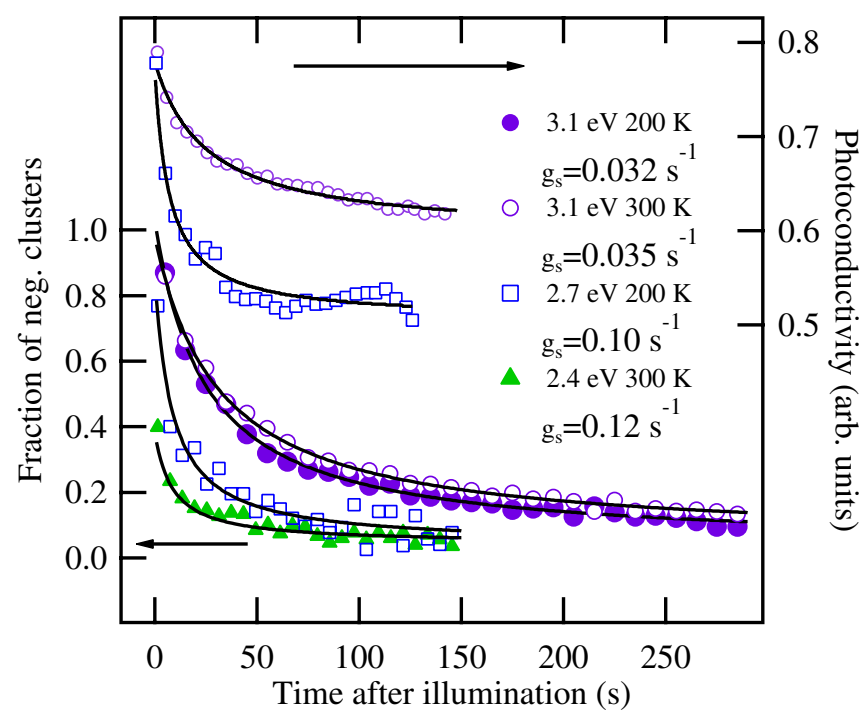

FIG. 3 (color online). The fraction of negative clusters at 200 and $300 \mathrm{~K}$ calculated from the trapping rate. The photoconductivity decay curves shown in the upper part were measured at RT with 2.7 and $3.1 \mathrm{eV}$ illumination using the van der Pauw configuration. The solid lines are fitted based on the model presented in the text. 


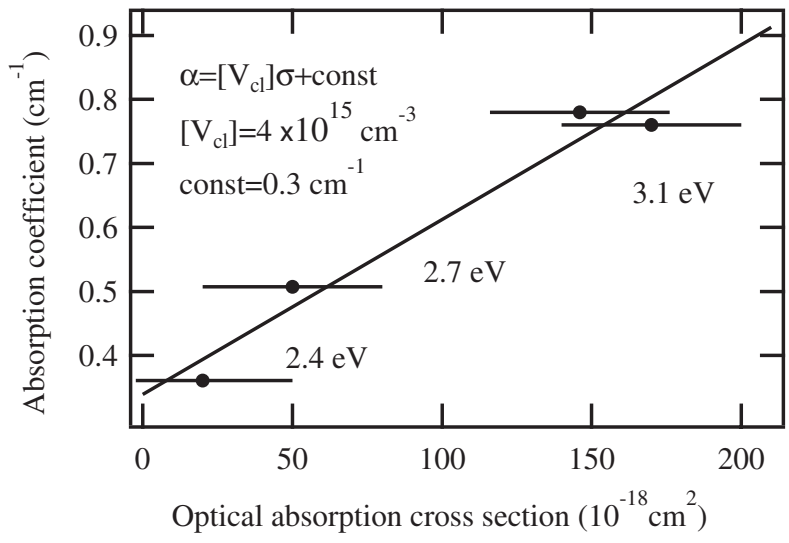

FIG. 4. The optical absorption coefficient $\alpha$ compared to the optical absorption cross section $\sigma$ at different photon energies. The slope of the fitted line is the vacancy cluster concentration $4 \times 10^{15} \mathrm{~cm}^{-3}$. The error bars are estimated from the scatter of the estimated cross section values.

reduced [8] compared to the untreated brown diamond studied here, verifying that the determined constants are independent of the defect concentration. The good correlation shows that electron photoexcitation from the valence band to the energy levels of the vacancy clusters is the main contributor to the optical absorption observed in brown type IIa diamond. Theoretical calculations [25] predict that clusters of vacancies produce a continuum of states in the gap, with the density of states increasing with the distance from the valence band, supporting this assignment.

We performed photoconductivity measurements in order to verify that photoexcited electrons indeed originate from the valence band. The samples showed no dark conductivity, while illumination resulted in a well measurable current through the samples (with $20 \mathrm{~V}$ bias). Based on earlier reports on Hall measurements under sub-band-gap photoexcitation, the photoconductivity in natural type IIa diamond is due to photogenerated holes [26]. In order to compare the diamond photoconductivity and the photoexcitation of vacancy clusters, the decay of photoconductivity was also measured.

The photoconductivity decay curves are shown in Fig. 3 for 3.1 and $2.7 \mathrm{eV}$ illumination. During the first $100 \mathrm{~ms}$ after switching off the illumination, the decay of the photocurrent is very rapid. After that, the behavior is in excellent agreement with the rates $g_{s}$ obtained from the positron measurements as a function of both photon flux and excitation wavelength. Also, the photoconductivity decay rates are rather insensitive to the illumination flux, suggesting that the average capture cross section for the holes indeed decreases as the photon flux is increased.

Our simple model does not to take into account the possible excitation of electrons from the energy levels of the vacancy clusters into the conduction band. This is a possibility at least at $3.1 \mathrm{eV}$. However, it seems that the optical absorption cross section for this process is small enough for this effect to be negligible. We wish to point out that the exact charge state of the vacancy clusters cannot be estimated based on the decay rate of the optical excitation, as the measurement is primarily sensitive to the difference between neutral and negative charge states. However, this does not affect the estimate of the optical absorption cross section, as the same "effective" transition rate back to neutral affects both the decay measurement and the steady-state measurements.

As natural diamond is known to contain several defects trapping charge carriers even in its purest form [27], it is in principle possible that the photogenerated carriers could get trapped at other traps than the vacancy clusters. Furthermore, the electrons that are photoexcited into the vacancy cluster states could in principle be originating from donor-character defect levels (such as nitrogen [28]) either directly or through the conduction band, instead of direct excitation from the valence band. These effects seem, however, unlikely as the positron data and the photoconductivity data transients interpreted within our simple model match irrespective of photon flux or wavelength. In addition, measurements performed down to $45 \mathrm{~K}$ (not shown) give the same results as those presented here, suggesting that no shallow traps for holes are involved in the recombination process.

In conclusion, we have developed a method based on positron annihilation spectroscopy that allows us to study the time-dependent relaxation of photoexcited vacancyrelated deep level states. The method can be applied to semiconductors and insulators where the charge state of vacancy defects can be manipulated by illumination. Importantly, the method is based on the direct detection of the excited state instead of, e.g., light emission from the relaxation process. To demonstrate the method, we apply it to natural type IIa diamond and propose a simple model to analyze the behavior of the carriers and defect levels in this material. The results suggest that both the photoconductivity and the brown color in type IIa diamond originate from the vacancy clusters. Finally, based on our simple model, we can estimate the vacancy cluster concentration as $4 \times 10^{15} \mathrm{~cm}^{-3}$ without direct knowledge of the positron trapping coefficient. It should be noted that the long decay time (hundreds of seconds) makes the experiment quite straightforward, but the time scale can be shortened to the millisecond regime with the present setup. Even shorter time scales (down to nanoseconds) can be achieved by employing a pulsed positron beam [29], entering the regime of, e.g., typical nonradiative recombination processes (that may be vacancy-related) in semiconductors for optoelectronics.

This work was partially funded by the MIDE program and the Academy of Finland. We wish to thank Dr. S. Novikov, Mr. E. Korhonen, and Ms. T. Kuittinen for assistance in experiments. 
*jussi-matti.maki@aalto.fi

[1] K. Saarinen, P. Hautojärvi, and C. Corbel, in Identification of Defects in Semiconductors, edited by M. Stavola (Academic, New York, 1998), p. 209.

[2] K. Saarinen et al., Phys. Rev. Lett. 79, 3030 (1997).

[3] F. Tuomisto et al., Phys. Rev. Lett. 91, 205502 (2003).

[4] R. Krause et al., Phys. Rev. Lett. 65, 3329 (1990).

[5] J. Mäkinen et al., Phys. Rev. Lett. 71, 3154 (1993).

[6] S. Arpiainen et al., Phys. Rev. B 66, 075206 (2002).

[7] V. Avalos and S. Dannefaer, Physica (Amsterdam) 340B342B, 76 (2003).

[8] J.-M. Mäki et al., J. Phys. Condens. Matter 21, 364216 (2009).

[9] J. Isberg et al., Science 297, 1670 (2002).

[10] G. A. J. Amaratunga, Science 297, 1657 (2002).

[11] P. W. May, Phil. Trans. R. Soc. A 358, 473 (2000).

[12] J.E. Butler et al., J. Phys. Condens. Matter 21, 364201 (2009).

[13] P. L. Stanwix et al., Phys. Rev. B 82, 201201 (2010).

[14] T. A. Kennedy et al., Appl. Phys. Lett. 83, 4190 (2003).

[15] S. Dannefaer, J. Phys. Condens. Matter 21, 175412 (2009).

[16] A. Uedono et al., J. Phys. Condens. Matter 11, 4109 (1999).
[17] V. Avalos et al., Diam. Relat. Mater. 14, 155 (2005).

[18] E. Fritsch, in The Nature of Diamonds, edited by G.E. Harlow (Cambridge University Press, Cambridge, England, 1998), p. 23.

[19] R. Jones, Diam. Relat. Mater. 18, 820 (2009).

[20] U. Bangert et al., J. Phys. Condens. Matter 21, 364208 (2009).

[21] F. Tuomisto et al., Phys. Rev. B 72, 085206 (2005).

[22] K. Saarinen et al., Phys. Rev. Lett. 70, 2794 (1993).

[23] M. J. Puska and R. M. Nieminen, Rev. Mod. Phys. 66, 841 (1994).

[24] P. Hautojärvi and C. Corbel, in Proceedings of the International School of Physics "Enrico Fermi," Course CXXV (IOS, Amsterdam, 1995), pp. 491-532.

[25] L. S. Hounsome et al., Phys. Rev. B 73, 125203 (2006).

[26] C. Saguy, E. Baskin, and R. Kalish, Diam. Relat. Mater. 14, 344 (2005).

[27] A. M. Zaitsev, Optical Propertis of Diamond: A Data Handbook (Springer, Berlin, 2001).

[28] A. T. Collins and E.C. Lightowlers, in Properties of Diamond, edited by J.E. Fields (Academic, London, 1979).

[29] A. Laakso, K. Saarinen, and P. Hautojärvi, Physica (Amsterdam) 308B-310B, 1157 (2001). 